\title{
Development of phased array ground penetrating radar for near surface exploration
}

\author{
Kaito KIKUCHI ${ }^{1}$, Hitoshi MIKADA ${ }^{2}$ and Junichi TAKEKAWA ${ }^{2}$ \\ ${ }^{1}$ Dept. of Civil and Earth Res. Eng., Kyoto University (Now at IBM Japan, Ltd.) \\ ${ }^{2}$ Dept. of Civil and Earth Res. Eng., Kyoto University
}

\begin{abstract}
We have confirmed the interaction among phased array antennas in our previous study. In this paper, we would like to discuss one of the powerful and practical schemes of using phased array antennas applied to near surface exploration using ground penetrating radar (GPR). GPR emits electromagnetic (EM) waves to the subsurface and to measure signals reflected back from buried anomalies for the estimation of the positions and shapes of the anomalies. Although phased array antennas could generate EM waves whose signal-to-noise ratio is superior to that emitted by the conventional GPR antenna, there has been a risk of the interaction among plural antennas could lower the signal-to-noise ratio due to electrical currents induced by magnetic field generated by the other antennas and it was necessary to confirm the level of the interaction or the unfavorable currents caused by the other antennas. We conduct a numerical simulation to evaluate the mutual coupling of each pair of phased array antennas to determine the best alignment of antennas in the design to maximize the level of emitted signals. We then conduct another simulation with heterogeneous ground model using our designed antenna in order to confirm the effectiveness of the phased array antenna in practice. Our results show that the phased array antenna enhances the signal-to-noise ratio compared to the conventional antenna and has a higher sensitivity to the targets located lateral to the survey lines with low interaction level between the neighboring two antennas. We conclude that the phased array antenna has a potential to be used as a new radar source for GPR.
\end{abstract}

\section{Introduction}

GPR is one of the practical EM tools for the near surface exploration. For example, buried pipes, land mines or electrical cables are the targets of the GPR survey. The near surface exploration before conducting the urban development is very important to prevent hazards such as subsidence or destruction of infrastructure. The conventional antennas loaded in GPR are dipole antenna, bow-tie antenna or aperture antenna ${ }^{1)}$. But the extent of the survey area is limited to beneath the survey lines because of the antenna radiation pattern. If we want to get more detailed information over the survey area, we have to draw large number of survey lines. However, it results in a huge amount of surveying time and cost.

Phased array antenna is one of the high directivity antennas and it is applied to GPR to transmit the EM waves to a desired direction. This antenna is made up of lots of antenna elements and controlling the phase and amplitude of the waves generated from each element changes the direction of EM waves. This system is well known for air radar systems such as military radar or weather radar. When it is used as a GPR source ${ }^{2)}$, we have to take into consideration in the interference of each element since the element of the array antenna should be closely arranged ${ }^{3)}$. In addition, we have to take into account in the heterogeneity of the subsurface, which may cause scattering or other complex phenomena of EM waves ${ }^{4}$.

In this study, we made two assumptions to develop a practical phased array GPR. First assumption is that we can minimize interaction effect between antenna elements by determining the suitable arrangement of the antenna elements considering the mutual effect. The second one is that we can carry out a scanning survey in a heterogeneous ground using our phased array GPR.

First, we design the dipole antenna as a conventional radar source. Then we calculate the mutual impedance of two antenna elements to evaluate the mutual coupling of phased array antenna and determine the best arrangement of antenna elements. We conduct some numerical simulations using a heterogeneous ground model including a concrete cube to compare the dipole antenna and phased array antenna. Finally, we conduct a numerical simulation utilizing the two kinds of antenna assuming an actual GPR survey. 


\section{Theory}

\section{(1) FDTD Method}

FDTD method is widely used for antenna analysis or EM scattering problems. The discrete formulation of Maxwell's equations is derived using Yee's staggered grid $^{5}$. It is one of the fastest and simplest ways to calculate synthetic EM wave fields. In this paper, C-PML absorbing boundary condition $^{6)}$ is implemented to the model edges to suppress artificial reflection waves.

\section{(2) Kirchhoff Migration}

Kirchhoff migration is applied to the observed data. By back-projecting the observed data using the Kirchhoff integral formulation shown in Schneider's paper $^{7)}$, the scattering points in the ground are emphasized.

\section{Antenna Setting}

\section{(1) Antenna Design}

To confirm the feasibility of phased array GPR, we compare a phased array antenna with a dipole antenna. In this paper, the both antenna has the length of $67.5[\mathrm{~cm}]$ and the phased array antenna is composed of $7 \times 7$ elements. The antenna elements are made of perfect electric conductor (PEC) and Gaussian pulse is inputted at the center of the antenna.

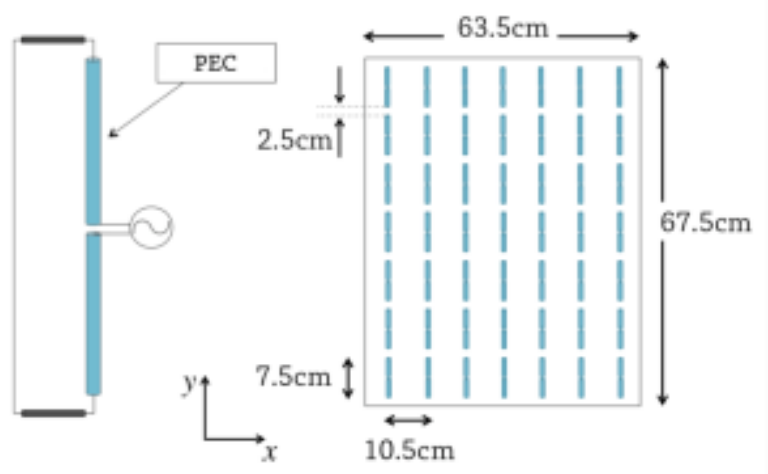

Figure 1: Dipole antenna (left) and phased array antenna (right).

\section{(2) Beamformer Method}

Beamformer method is one of the simple approaches to control the direction of EM waves. Delaying the incident time of each antenna of the array using (1), we can obtain the directivity of the radiated energy towards a desired direction.

$$
T_{0}=\frac{d \sin \theta}{v}
$$

where $d$ is the interval of each antenna and $v$ is the velocity of the EM waves in the ground. $\theta$ represents the deviation from the vertical direction.

\section{(3) Antenna Ringing}

Transmitting waves radiated from a dipole antenna are generally deformed by the multi-reflection at the both ends of the antenna. This deformation is called antenna ringing and it leads to undesired noises in GPR data.

In our simulation, we put the resistors $(150 \Omega)$ at the both ends and connect them with a wire in order to reduce the antenna ringing.

\section{(4) Antenna Mutual Coupling}

Since the elements of the phased array antenna are closely placed, the effect of antenna coupling cannot be disregarded. The amount of mutual coupling is evaluated by mutual impedance.

Impedance matrix is the inverse of admittance matrix, which is calculated from the equations shown in Luebbers and $\mathrm{Karl}^{8)}$.

$$
\begin{aligned}
& I_{1}(\omega)=V_{1}(\omega) Y_{11}+V_{2}(\omega) Y_{12} \\
& I_{2}(\omega)=V_{1}(\omega) Y_{21}+V_{2}(\omega) Y_{22}
\end{aligned}
$$

where $V_{1}, V_{2}$ are the input and output voltage in antenna 1 and antenna 2, respectively (Figure 2). $I_{1}$, $I_{2}$ are the output current. In this paper, two patterns of impedance matrices are calculated, broadside and collinear array (Figure2, 3).

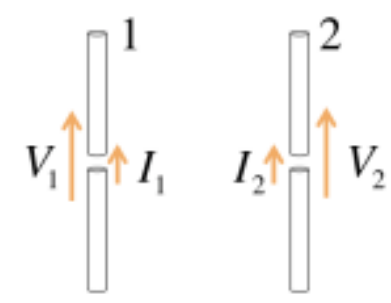

Figure 2: Broadside array antennas.
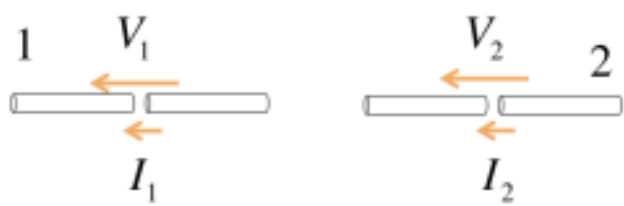

Figure 3: Collinear array antennas.

The mutual impedance is shown in Figure 4. The red line represents the mutual impedance of broadside array and the blue line represents that of the collinear array. Black stars are the intervals that 
we take, $10.5[\mathrm{~cm}]$ for broadside array and $2.5[\mathrm{~cm}]$ for collinear array.

We confirmed that the mutual effect for broadside array is stronger than the collinear array. Thus, it is important to reduce the mutual effect of the broadside array to determine the suitable arrangement of phased array antenna.

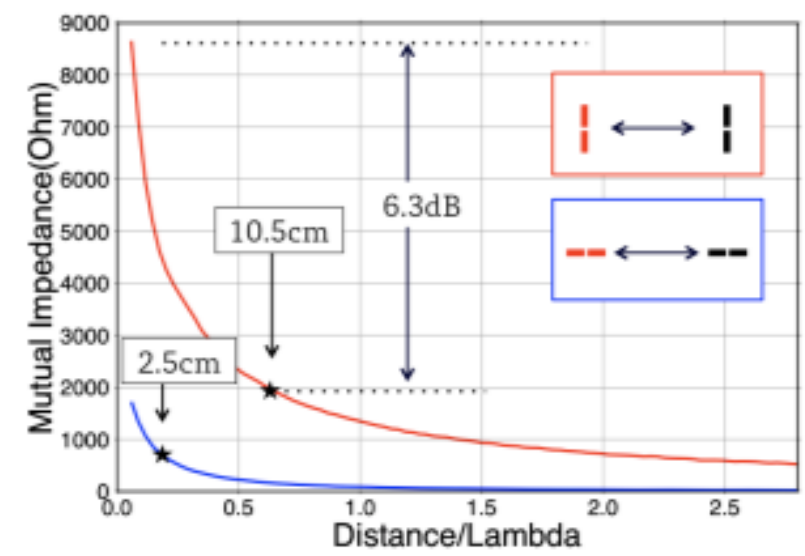

Figure 4: Mutual impedance of the array. (Red line: broadside array, Blue line: collinear array)

\section{Numerical Simulations}

\section{(1) Comparing two Antennas}

We conduct a numerical simulation using a heterogeneous ground model (Figure 5) to confirm the advantage of phased array antenna in GPR survey. Heterogeneity is expressed by water bodies and PEC bodies, which are distributed throughout the model. The main target is a concrete cube and it lies apart from the antenna. Two simulations are carried out using the dipole antenna and the phased array antenna. Both antennas are fixed and the phased array antenna radiates the energy towards the concrete cube.

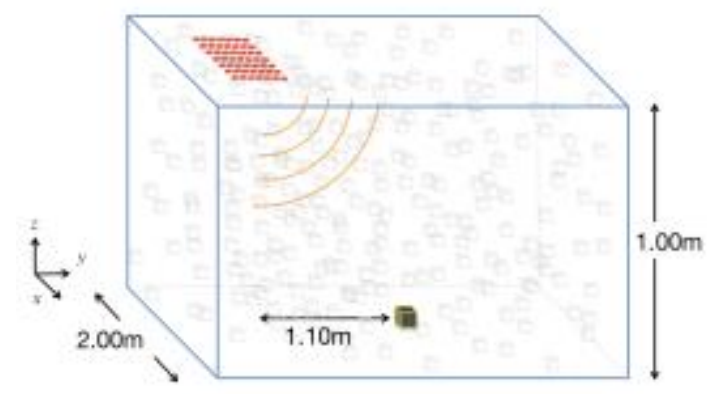

Figure 5: Ground model. Brown cube is concrete and transparent cubes are the water and PEC bodies.

Figure 6 shows the scattered waves observed by the GPR. The red and blue lines show the waveforms obtained by the phased array and the dipole antenna, respectively. The continuous vibrations are the noises coming from the heterogeneity. The strong wave around $3.0 \mathrm{e}-08$ [sec] is a scattered wave from the concrete cube.

Comparing the red and blue lines, the phased array antenna enhances the amplitude of a scattered waves from the concrete cube lying apart from the antenna and also dramatically enhances the signal-to-noise ratio.

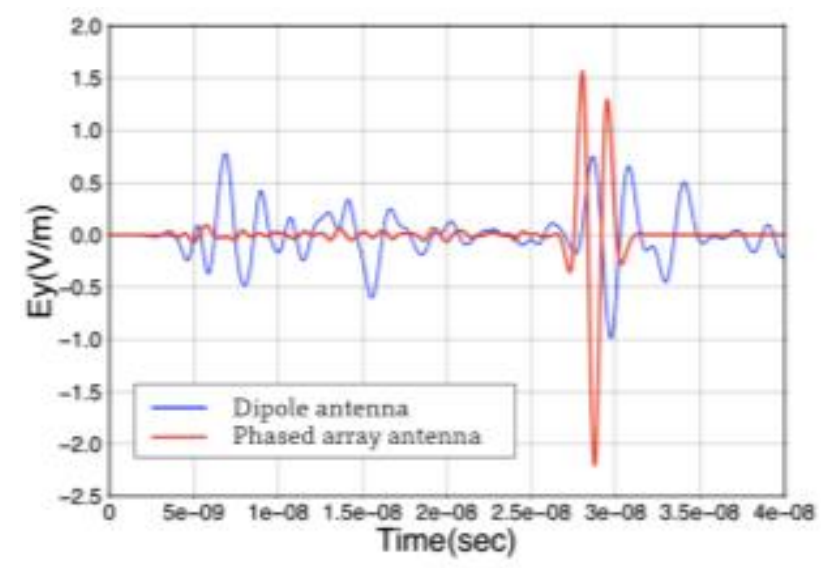

Figure 6: Comparison of scattered waves of dipole antenna and phased array antenna.

\section{(2) Migration results}

To confirm the usability of the phased array GPR under a practical condition, we conduct another numerical simulation assuming an actual GPR survey. Two concrete cubes are placed between two survey lines (Figure 7) and we move the two kinds of antenna along the lines ( $x$-axis). Phased array antenna radiates the EM waves to 7 different directions.

We apply Kirchhoff migration to the observed data for the estimation of the ground model. The migration is applied inside the green cube.

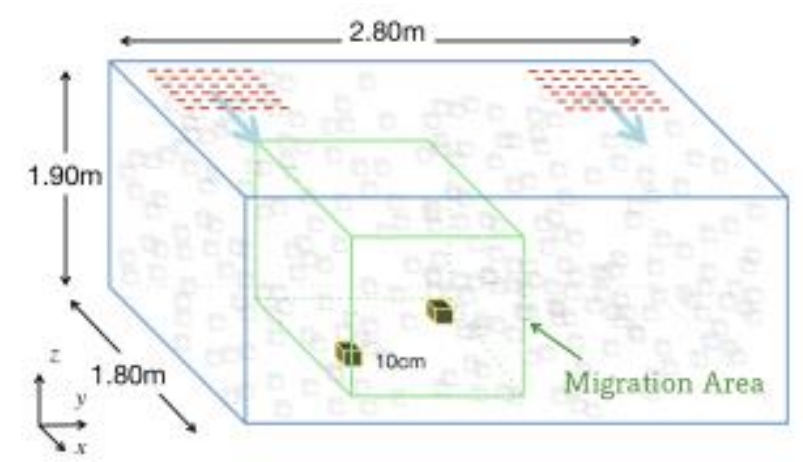

Figure 7: Ground model. Blue arrows show the direction that antennas are moving.

Figure 8 and 9 show the migration results obtained by the dipole antenna and the phased array antenna, respectively. The strong signals (red) stand for the estimated position of the concrete cubes. 
Comparing the migration results, we comfirmed that the phased array antenna has an advantage in enhancing the signal-to-noise ratio. In addition, the phased array antenna properly distinguish the two concrete cubes while the dipole antenna couldn't.

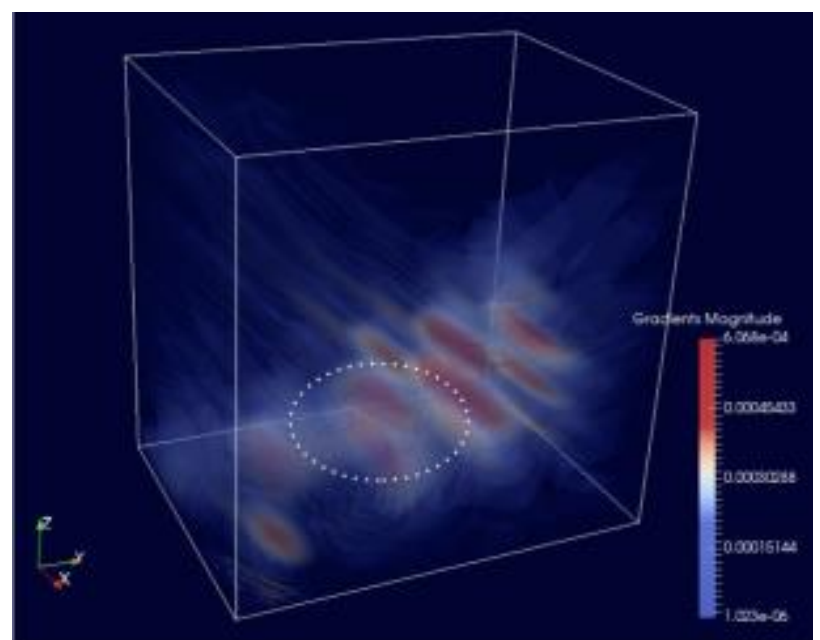

Figure 8: Subsurface image obtained by dipole GPR.

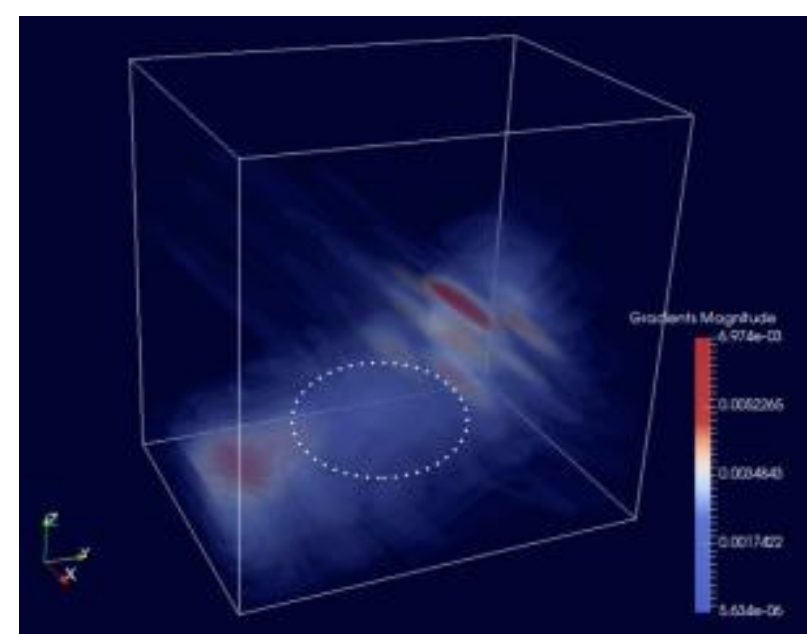

Figure 9: Subsurface image obtained by phased array GPR.

Figure 10 shows the cross sections in $y z$-plane obtained by phased array antenna. We confirmed that the signals converge to an actual position with $0.58 \%$ error.

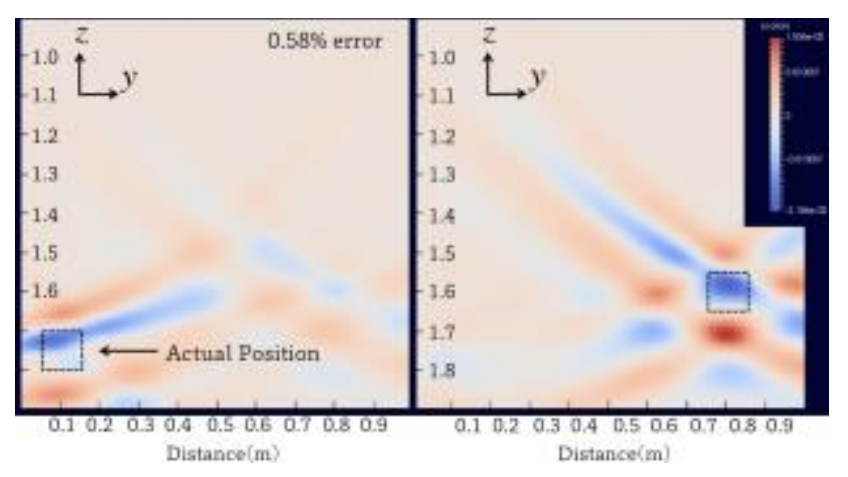

Figure 10: Cross section obtained by phased array antenna

\section{Conclusion}

In this paper, we made two assumptions to develop a practical phased array GPR. The first assumption is that we can minimize the effect of antenna interaction by determining the suitable arrangement of the antenna element considering the antenna mutual effect. We calculate the mutual impedance to evaluate the mutual effect of array antenna. We confirmed that the mutual coupling of the broadside array is stronger than that of the collinear array, so that the interval of broadside array has to be as long as possible. The second assumption is that we can conduct a scanning survey in a heterogeneous ground model. We carried out a numerical simulation using our designed dipole and phased array antenna. Our results show that the phased array antenna has an advantage in enhancing the signal-to-noise ratio. Also, the phased array antenna distinguished the two concrete cubes located out of the survey line.

We conclude that our developed phased array GPR can be used as an angular-scanning imaging tool, which covers greater extent of survey area and enables us to save the survey cost.

\section{REFERENCES}

1) Sato, M., and Toshioka, T., 1998 "Methodology, Chapter 7 GPR, Butsuri Tansa Handbook" in Society of Exploration Geophysics of Japan (ed.).

2) Kikuchi, K. K., Mikada, H., \& Takekawa, J. T., 2016 "Applicability of Phased Array Antenna to Ground Penetrating Radar for Subsurface Imaging Below Surface Obstacles" In 78th EAGE Conference and Exhibition 2016.

3) Adachi, S., 1970 "Practical use of array antenna and its problems" television, 24(5), 333-340.

4) Das, U., Boer, H. J., \& van Ardenne, A., 2003 "Phased array technology for GPR antenna design for near subsurface exploration" In Advanced Ground Penetrating Radar, 2003. Proceedings of the 2nd International Workshop on (pp. 30-35). IEEE.

5) Yee, K. S., 1966 "Numerical solution of initial boundary value problems involving Maxwell's equations in isotropic media." IEEE Trans. Antennas Propag, 14(3), 302-307.

6) Roden, J. A., \& Gedney, S. D., 2000 "Convolutional PML (CPML): An efficient FDTD implementation of the CFS-PML for arbitrary media." Microwave and optical technology letters, 27(5), 334-338.

7) Schneider, W. A., 1978 "Integral formulation for migration in two and three dimensions" Geophysics, 43(1), 49-76.

8) Luebbers, R., \& Kunz, K., 1992 "Finite difference time domain calculations of antenna mutual coupling" IEEE Transactions on Electromagnetic Compatibility, 34(3), 357-35. 